\title{
Granger causality is designed to measure effect, not mechanism
}

\author{
Adam B. Barrett* and Lionel Barnett \\ Department of Informatics, Sackler Centre for Consciousness Science, University of Sussex, Brighton, UK \\ *Correspondence: adam.barrett@sussex.ac.uk \\ Edited by: \\ Marc-Oliver Gewaltig, Ecole Polytechnique Federale de Lausanne, Switzerland \\ Reviewed by: \\ Marc-Oliver Gewaltig, Ecole Polytechnique Federale de Lausanne, Switzerland \\ Athanassia Chalimourda, Ecole Polytechnique Federale de Lausanne, Switzerland
}

\section{A commentary on}

Causality analysis of neural connectivity: critical examination of existing methods and advances of new methods

by Hu, S., Dai, G., Worrell, G. A., Dai, Q., and Liang, H. (2011). IEEE Trans. Neural Netw. 22, 829-844.

In their recent paper, $\mathrm{Hu}$ et al. (2011) make the claim that Granger causality (GC) does not capture how strongly one time series influences another. Given the sizeable literature on GC, this claim could be considered radical. We examined this claim, and found that it is based essentially on semantics. Hu et al. (2011) would like a measure of causal interaction to explicitly quantify an underlying causal mechanism, and point out that GC values do not consistently reflect the relative sizes of explicit interaction coefficients in a corresponding generative model. However, GC is, by design and purpose, not interested in this. Rather, it is a measure of causal effect, namely the reduction in prediction error when the causal interaction is taken into account, as compared to when it is ignored. [According to one version of neuroscience terminology (Friston, 2011), which attempts to draw a distinction between the different conceptions of connectivity, GC measures of causal effect yield directed "functional connectivity" maps when applied to neuroimaging data. In contrast, "effective connectivity" maps represent the effective mechanism generating the observed data, and provide interaction coefficients. Neither functional nor effective connectivity representations necessarily map univocally onto the underlying anatomical (structural) connectivity.]

Multiple properties of GC make it an elegant measure of causal effect. It satisfies crucial symmetry properties, including that GC from $Y$ to $X$ is invariant under rescalings of $Y$ and $X$, as well as the addition of a multiple of $X$ to $Y$, consistent with the measuring of independent predictive information about $X$ contained in Y (Geweke, 1982; Hosoya, 1991; Barrett et al., 2010). Such transformations do however, change the relative magnitudes of regression coefficients, thus it is not possible to simultaneously measure causal mechanism and causal effect. Further, for the case of Gaussian variables, GC is equivalent to transfer entropy, enabling an explicit interpretation in terms of Shannon information flow (Barnett et al., 2009). The GC from one multivariate variable to another multivariate variable has a decomposition into the sum of independent contributions from each predictor to each predictee [equation 18 in Barrett et al. (2010)]. The same defence of GC applies in the frequency domain, with spectral GC from $Y$ to $X$ at frequency $f$ capturing the proportion of power of $X$ at frequency $f$ that results from its interaction with $Y$ (Geweke, 1982). The fact that time domain GC is the mean spectral GC over all frequencies up to the Nyquist frequency provides further justification.

As far as statistical inference is concerned, for the reasons above, GC can indeed be used to compare the magnitude of causal interactions between different sets of time series. Contrary to Hu et al.'s (2011) interpretation, the fact that some regression coefficients contribute more to GC than others (and some not at all) is actually an indication that GC analysis adds to our understanding of a system, even when the generative model is known a priori. A further pragmatic advantage of the GC method is that, in sample, time-domain GC asymptotically follows distributions that are known analytically (chi-squared family), thus facilitating hypothesis testing (Geweke, 1982).

Note on Redundancy: A specific argument Hu et al. (2011) make against GC comes from the behaviour of the measure for the system given by their equation 10. Hu et al. (2011) compute the GC from $X_{2}$ to $X_{1}$ to be zero when the residual $\eta_{2}$ associated with $X_{2}$ has zero variance, but claim that a measure of causal influence should be non-zero in this case. However, in this case, $X_{2}$ is a redundant variable, being fully determined by the past of $X_{1}$, and therefore, does not influence $X_{1}$ once the past of $X_{1}$ is taken into account. In other words, $X_{2}$ has no independent causal influence on $X_{1}$ and it is therefore, entirely consistent for GC to be zero in this case.

GC is not a perfect measure for all stochastic time series: if the true process is not a straightforward multivariate autoregressive process with white-noise residuals, then it becomes only an approximate measure of causal influence. In each real-world scenario, discretion is required in deciding if confounds such as nonlinearity and correlations in the noise are mild enough for the measure to remain applicable. In these scenarios, it can be useful to consider a range of different measures such as Phase Slope Index (Nolte 
et al., 2008), Partial Directed Coherence (Baccalá and Sameshima, 2001), and the Directed Transfer Function (Kaminski and Blinowska, 1991; Kaminski et al., 2001).

In summary, GC measures causal effect in a clear and unambiguous way on stationary multivariate autoregressive processes. We believe that the measure is rightly being widely applied in neuroscience as a measure of directed functional connectivity whenever such models provide a reasonable fit to data. Hu et al.'s (2011) "new causality" compares regression model coefficients rather than prediction errors, and is therefore a measure of causal mechanism. New causality sets out to achieve a different aim from GC, and the divergence of the two measures is not a problem.

\section{ACKNOWLEDGMENTS}

We thank Anil Seth for providing valuable suggestions on a draft of this piece. Adam B. Barrett is funded by EPSRC grant EP/G007543/1. Lionel Barnett is supported by the Dr. Mortimer and Theresa Sackler Foundation.

\section{REFERENCES}

Baccalá, L. A., and Sameshima, K. (2001). Partial directed coherence: a new concept in neural structure determination. Biol. Cybern. 84, 463-474.

Barnett, L., Barrett, A. B., and Seth, A. K. (2009). Granger causality and transfer entropy are equivalent for Gaussian variables. Phys. Rev. Lett. 103. doi: 10.1103/PhysRevLett.103.238701

Barrett, A. B., Barnett, L., and Seth, A. K. (2010). Multivariate Granger causality and generalized variance. Phys. Rev. E 81. doi: 10.1103/PhysRevE. 81.041907

Friston, K. J. (2011). Functional and effective connectivity: a review. Brain Connect. 1, 13-36.

Geweke, J. (1982). Measurement of linear dependence and feedback between multiple time series. J. Am. Stat. Assoc. 77, 304-313.

Hosoya, Y. (1991). The decomposition and measurement of the interdependency between secondorder stationary processes. Probab. Theory Relat. Field 88, 429-444.

Hu, S., Dai, G., Worrell, G. A., Dai, Q., and Liang, H. (2011). Causality analysis of neural connectivity: critical examination of existing methods and advances of new methods. IEEE Trans. Neural Netw. 22, 829-844.

Kaminski, M., and Blinowska, K. J. (1991). A new method of the description of the information flow in brain structures. Biol. Cybern. 65, 203-210.

Kaminski, M., Ding, M., Truccolo, W. A., and Bressler, S. L. (2001). Evaluating causal relations in neural systems: Granger causality, directed transfer function and statistical assessment of significance. Biol. Cybern. 85, 145-157.

Nolte, G., Ziehe, A., Nikulin, V., Schlögl, A., Krämer, N., Brismar, T., et al. (2008). Robustly estimating the flow direction of information in complex physical systems. Phys. Rev. Lett. 100. doi: 10.1103/ PhysRevLett.100.234101

Received: 25 March 2013; accepted: 08 April 2013; published online: 25 April 2013.

Citation: Barrett AB and Barnett L (2013) Granger causality is designed to measure effect, not mechanism. Front. Neuroinform. 7:6. doi: 10.3389/fninf.2013.00006 Copyright (c) 2013 Barrett and Barnett. This is an open-access article distributed under the terms of the Creative Commons Attribution License, which permits use, distribution and reproduction in other forums, provided the original authors and source are credited and subject to any copyright notices concerning any thirdparty graphics etc. 